\title{
Polysèmes
}

Revue d'études intertextuelles et intermédiales

\section{L'immobilité vive, ou « une petite étoile à la vitre du texte »}

Intense Immobility or "a Small Star on the Window Pane of the Text"

\section{Liliane Louvel}

\section{OpenEdition}

Journals

Édition électronique

URL : http://journals.openedition.org/polysemes/2299

DOI : 10.4000/polysemes.2299

ISSN : 2496-4212

Éditeur

SAIT

Référence électronique

Liliane Louvel, «L'immobilité vive, ou « une petite étoile à la vitre du texte » », Polysèmes [En ligne],

18 | 2017, mis en ligne le 30 novembre 2017, consulté le 20 avril 2019. URL : http://

journals.openedition.org/polysemes/2299; DOI : 10.4000/polysemes.2299

Ce document a été généré automatiquement le 20 avril 2019.

Polysèmes 


\title{
L'immobilité vive, ou « une petite étoile à la vitre du texte »
}

Intense Immobility or "a Small Star on the Window Pane of the Text"

\author{
Liliane Louvel
}

1 «L'immobilité vive» que l'on nous donne à penser ici repose sur un oxymore, un paradoxe, l'alliance de contraires habituellement opposés comme dans la dichotomie, classique au sein des études artistiques, entre la vie et la mort, l'amour et la haine, le temps et l'espace, etc. - une alliance de contraires, ce qui en contrepartie n'est pas le cas du texte et de l'image.

2 Pour nous aider à penser "l'immobilité vive ", allons donc voir du côté de l'antithèse aussi. À « l'immobilité vive » s'opposerait alors « la mobilité morte ». Le fait d'être mobile, susceptible de changements, est ce qui caractérise le vivant. La discipline scientifique récemment re-nommée "sciences de la vie [la biologie] et de la terre [la géologie]» s'intéresse aux êtres humains mais aussi aux animaux, aux plantes, aux roches... - ce que l'on nommait autrefois « les sciences naturelles ». On peut donc en conclure que le vivant relève de la nature, contrairement à ce qui est fabriqué, produit d'une industrie non naturelle. Ce qui ramène "la mobilité morte", contraire (et je dirais, pour employer un terme pictural, « pendant ») de « l'immobilité vive », du côté de « la nature morte ». Dans l'oxymore de départ, la juxtaposition des termes, et donc la proximité des notions qui implique l'idée de simultanéité, prête à questionnement. Pourrait-on être immobile et vif ? mobile et mort ? L'art dans sa liberté constitutive nous autoriserait à le penser.

Dans La Chambre claire, le terme d'«immobilité vive» est utilisé pour rapprocher la photographie et le haïku. Tous deux sont, pour Barthes, de l'ordre du punctum, de «l'indéveloppable» de la "photo pointée " (Barthes 81), de l'effet «instantané » et frappant, du côté de l'évidence singulière qui laisse sans voix :

Ceci rapproche la Photographie (certaines photographies) du Haïku... Tout est

donné, sans provoquer l'envie ou même la possibilité d'une expansion rhétorique.

Dans les deux cas on pourrait, on devrait parler d'une immobilité vive : liée à un 
détail (un détonateur), une explosion fait une petite étoile à la vitre du texte ou de la

photo : ni le Haïku ni la photo ne font « rêver ». (Barthes 81-82, je souligne)

C'est donc l'effet saisissant de l'image (visuelle, écrite) qui inscrit sa fulgurance dans un moment où se révèle «quelque chose ", qui fait que « la photo n'est plus quelconque ». Nous voilà du côté de la réception, de l'effet, de l'avènement.

Lorsque Barthes écrit que le punctum ne provoque pas l'envie d'une expansion rhétorique, nous voilà a priori hors du domaine de l'ekphrasis. «L'immobilité vive » me poindrait sans me donner désir de parole. Voilà qui peut sembler paradoxal en ce qui concerne l'imageen-texte et le rapport texte/image qui forcément relèvent de l'expansion rhétorique, qui traduit en mots ce qui « me point ». Il faudra revenir là-dessus.

Mais continuons notre interrogation sémantique. Si, au lieu du contraire de « l'immobilité vive », on cherche le semblable ailleurs, comme dans d'autres langues, on trouverait en anglais, outre " intense immobility ", "vivacious stillness ", " living stillness », ce qui donne, en inversant les termes en chiasme, «still life », c'est-à-dire la traduction de « nature morte ». Où l'on retrouve la nature qui tient la place de la « vie ». «L'immobilité vive » serait-elle donc d'abord l'autre nom de la « vie/nature morte »? Le domaine d'une nature immobile donc marquée par la mort? On se rappelle que Barthes dans sa fameuse analyse des pâtes Panzani, dans « Rhétorique de l'image ", écrit still living pour still life. Il faisait ainsi un intéressant lapsus de traduction, "still living» pouvant signifier au contraire « encore en vie » et non au repos, morte, immobile ${ }^{1}$.

6 Ceci n'enlève rien à la subtilité de la démonstration des premières heures de la sémiotique de l'image, mais montre à quel point l'image «lue » comme un ensemble de signes - ce qui faisait fureur dans les années soixante -, et non en termes propres au medium, relevait de l'inféodation, certains diront de la domination, de l'impérialisme, du rapport dit intersémiotique auquel on préfère intermédial, en termes de «genre/ gender » aussi.

7 Immobilité/mort/mobilité/vie pourraient même s'inscrire dans un carré sémiotique en une roue infernale où le sens n'en finirait pas de passer. La mobilité vive et l'immobilité de la mort sont des lieux communs. Que l'on change les adjectifs de place et le sens équivoque joue du paradoxe oxymoronique. Nous revoilà au début de la démonstration. Le mouvement ne s'accommode pas de la mort (sauf dans le fantastique) ni de l'immobilité de la vie, car le temps implique le changement, soit que tout bouge à chaque seconde, inexorablement : voir à ce propos les œuvres de Sam Taylor-Wood. Seule la mort fige le vif dans le cadre doré de la porte où, dans The Portrait of a Lady, Isabel Archer s'encadre pendant quelques secondes, les secondes où elle coïncide avec son portrait, dans la lumière de la fenêtre de la représentation (voir Gérard Wajcman).

\section{Premières questions}

Le rapport texte/image vu par les fidèles de Lessing depuis des lustres impliquerait le croisement des arts de l'espace et des arts du temps. Le texte, dans le déroulement temporel de sa lecture, semble s'arrêter dans une ekphrasis pour laisser place à une image qui relèverait de l'espace. Rien de plus faux, puisque l'image-en-texte est encore du texte ("text-time»), du déroulement, soit du texte dont l'effet est autre, même si l'impression d'arrêt sur image de la lecture (de fait celui de la narration, "story time ») implique un changement de rythme et d'appréhension dont l'étude relève des sciences 
cognitives. L'effet de lecture et "l'événement de lecture ", voire de "voyure ", sont à interroger lorsque l'on évoque « l'immobilité vive » de la Photographie, du Haïku.

Le rapport texte/image implique d'emblée une position instable puisqu'il s'agit d'un rapport et non pas d'un ordre fixe. Dans l'ekphrasis par exemple, l'oscillation est constitutive de l'émergence de l'image qu'elle soit fictive (ou «notional», selon Heffernan) ou réelle (« real »). Une image se lève du texte, ainsi que le préconisait Daniel Arasse qui, face à un tableau (la Madone de Dresde de Raphaël, dans son cas), conseillait de laisser l'image se lever. Mais dans le dispositif, ceci se passe entre deux media, entre texte et image, ainsi que dans l'esprit du lecteur qui doit accepter de n'être qu'une sorte d'écran de réception, le lieu d'un "événement de lecture ». C'est ce que décrit Louis Marin à propos de sa rencontre inopinée avec les dessins de Stendhal présents dans les manuscrits de Vie de Henri Brulard, qui «a provoqué [en lui] un petit ébranlement, un satori » dirait Barthes (81).

L'oscillation constitutive du rapport texte/image est aussi ce que j'ai appelé le lieu de l'émergence du tiers pictural, cette apparition entre deux, comme nous le verrons.

«L'immobilité vive » combinerait alors l'apparente fixité de l'image, comme une fois pour toutes inscrite dans son cadre, avec la mobilité qui lui serait conférée par le texte qui l'animerait, lui donnerait vie, l'inscrirait sur l'axe du temps et de l'histoire. C'est ce que l'on trouve dans les textes gothiques ou fantastiques lorsque l'image s'anime et se narrativise. Les personnages sortent ou entrent dans les tableaux, comme dans le conte oriental de M. Yourcenar. Les femmes y meurent comme dans «The Oval Portrait» de Poe lorsque leur substance y passe vampirisée ; ou encore le tableau se métamorphose et porte les traces du changement moral du personnage, le paradigme en étant The Picture of Dorian Gray. Le tableau ou la photographie révèlent aussi une histoire, comme dans Martha Peake de Patrick McGrath, The Invention of Solitude de Paul Auster, ou encore Blindfold de Siri Hustvedt.

Mais les choses sont plus complexes, on s'en doute, car si le texte peut figer l'image en lui donnant un lieu où se poser, il est alors le lieu d'une immobilité et non plus celui d'une vie. Les "tableaux vivants" sont un parfait exemple d'«immobilité vive» réalisée. Lorsque Lily Barth dans The House of Mirth apparaît sur scène et prend la pose (Edith Wharton, 1905), elle coïncide avec le portrait de Mrs Lloyd peint par Reynolds, elle " est " le portrait et ainsi se donne une mort symbolique sous les yeux de ses admirateurs, Selden en particulier. «L'explosion » de son apparition est en même temps une machine de mort. Comme si elle était enterrée vivante dans la représentation d'elle-même comme double de l'image - ce qui ne manquera pas de se passer à la fin du roman, lors de la dernière scène dépeinte avec les outils de la peinture, sous les yeux du même Selden.

Les écrans informatiques qui font apparaître les images et les textes comme images sont le lieu du tremblement de leurs pixels qui, vivement, vacillent et produisent une image susceptible de disparaître à tout moment, comme douée de vie et donc de mort. Ils font changer le paradigme. Rappelons que Walter Benjamin se penchant sur l'émergence de «l'œuvre d'art achevée » avait reconnu la nécessité de trois facteurs: la technique qui évolue, la tradition qui s'épuise à produire des effets, la société qui se transforme et entraine dans le mode de réception un changement qui favorise la nouvelle forme d'art. La demande nouvelle pousse alors au dépassement.

En fait toute forme d'art achevée se trouve au croisement de trois lignes évolutives. En premier lieu, la technique prépare laborieusement l'apparition d'une forme d'art déterminée. En second lieu, les formes d'art traditionnelles, à certains stades de 
leur développement, travaillent péniblement à produire des effets qui, plus tard, seront obtenus sans contrainte par la nouvelle forme d'art. En troisième lieu, des transformations, souvent peu apparentes de la société, entraînent dans le mode de réception un changement qui favorise la nouvelle forme d'art. Chaque fois qu'apparaît une demande foncièrement nouvelle, elle dépasse son propos. (Benjamin 172-173)

Car si le texte peut sembler fixer, comme épingler l'image à l'instar d'un papillon sous verre, l'image, elle, apporte vie au texte dans l'éblouissement de sa vision, l'écarquillement de l'œil, l'ouverture émerveillée d'un instant de vie donné à la vue. Lorsque l'œil du texte s'ouvre à l'image, le rythme du texte change, le temps semble se distendre pour laisser place à l'image qui va occuper une zone de texte mais aussi le fond de l'œil du lecteur. Dans la « voyure ", le lecteur contemple alors ce qui n'est pas donné sous ses yeux mais se donne à voir néanmoins dans le tremblé du tiers pictural. Il faut une dose d'immobilité pour distinguer l'image et en distinguer les détails, mais ce faisant le temps passe, le lecteur/voyeur/spectateur saisit les différentes parties du donné à voir qui prend vie le temps de la lecture. L'immobilité serait-elle comme une condition de la vie ici?

\section{La nature morte}

La "nature morte ", revenons à elle, exemple pictural de la combinaison entre les deux paradigmes de la vie et de la mort, cette «still life » ou vie immobile, interroge le terme français qui associe nature à mort. Non pas vie, mais nature. Pourquoi ? Qu'y a-t-il de naturel dans ces représentations d'objets, de fruits, de fleurs, de livres, de chandelles fumantes, voire de crânes, comme dans la Vanité, cas particulier de nature morte, plus morte que vive. "La nature morte " proprement dite, qui ne relève peut-être pas de l'immobilité vive de Barthes (toute pleine de force, de fulgurance, de vie), est la représentation de ce qui ne bouge pas/plus. Située au bas de la hiérarchie des genres en peinture, elle restera longtemps confinée à un but décoratif, jusqu'à Chardin qui, osant les reflets nacrés de ses raies, scandalisera par leur ressemblance et leur trivialité « au vif semblant » (selon France Borel). Avec les bouquets ou les fruits contaminés par le temps, la déchéance et la mort, avec les papillons qui l'animent en sortant de leur chrysalide abandonnée sur le tapis de table, la nature morte indique le temps de la métamorphose à l'œuvre. Lorsque les fruits déjà se gâtent, que les citrons s'offrent à demi-ouverts ou à moitié épluchés, que les fleurs se fanent et que les premiers pétales tombent au pied du vase, alors le gibier mort exposé en trophée rappelle que la nature morte est vraiment un prélèvement d'objets vifs de la nature (fruits, fleurs, animaux), engagés dans un processus mortel irréversible. Memento mori aussi.

Comment alors montrer le processus et non seulement le suggérer ? Comment l'inscrire dans un éclat de temps ? À la National Gallery, avec A Little Death (2002), Sam Taylor-Wood a apporté une réponse en exposant la vidéo d'un lièvre mort, accroché par une patte, la tête pendante dans la plus pure tradition des trophées de chasse. Exemple de "nature » morte, de vie immobile dans la mort. La pièce est une vidéo dont le dispositif cite les œuvres anciennes: mais l'intérieur du «tableau »/écran s'agite et vit, montrant le processus de décomposition à l'œuvre. En accéléré, le spectateur peut suivre le travail des vers et des mouches qui vident le lièvre de sa substance ne laissant plus à la fin du processus qu'une peau vide et flasque accrochée au clou du mur. L'immobilité vive du gibier vivement re-montée est animée du travail des charognards qui prolongent ainsi 
l'activité de l'animal réputé si rapide, d'un autre type de vie, faisant échapper la dépouille à l'immobilité («stillness») de sa vie arrêtée. À noter, l'immobilité et la permanence paradoxale du fruit (une pêche?) situé au premier plan à gauche. De même, lorsque Taylor-Wood filme une coupe de fruits, intitulée Still Life (2001), en train de se décomposer, jusqu'à n'être plus qu'un tas mou et grisâtre aux contours indistincts, elle place un stylo à bille au premier plan qui, lui, reste identique à lui-même. Enfin, à la demande de la National Gallery, elle a réalisé en 2002 un portrait de David Beckham, film d'une véritable immobilité vive : David Beckham Sleeping...

Un exemple de nature morte mise en texte/image que j'ai déjà pu étudier s'intéresse surtout à des objets manufacturés, au contraire. Et pourtant, il entre dans la catégorie des «natures mortes». Je veux parler de la description du chapitre 11, "Containers », de Gabriel Josipovici dans Goldberg: Variations (2002).

Dans ce chapitre, le lecteur découvre une suite de mots décrivant des objets, jarres, étagère, placard, bouteilles, relevant donc du contenant, qui donnent au lecteur/ spectateur l'impression qu'il s'agit bien d'un tableau. Mais rien ne l'indique. Il faut donc se muer en détective et aller voir ailleurs. Se pose la question de ce qui dans le texte attire l'attention sur le côté pictural de la description qui, ce sera confirmé plus loin, est bien une ekphrasis.

Les objets sont alignés au gré du visionnement de l'œil qui balaie, se déplace, lui, selon le regard du focalisateur - à la différence des objets statiques, et du narrateur. Pour Bernard Noël, «ce qui bouge dans la peinture c'est uniquement le regard du spectateur » (Noël 13). Le texte de Josipovici semble vouloir se mouler sur une image en présentant les objets avant les verbes de repérage. Ils sont présentés comme "visés " par un regard et organisés par lui en suivant «le parcours du regard», son balayage imposé par le dispositif du tableau. Peut-être là avons-nous un premier élément de réponse : un tableau est composé (et non un paysage «naturel») et donc il impose au spectateur un certain « ordre assemblé ». Le regard est conduit, cela se lit et se prouverait. La description, grâce à certains adverbes, adjectifs, verbes ou noms, comme le terme de "surface ", suggère davantage un espace en deux dimensions qu'en trois dimensions, un espace dont on ne peut faire le tour. Le regard est «affecté » par l'image : d'abord attiré, par exemple, par un objet ou par un visage, dont les yeux convoquent fixement le spectateur («admonitor »). Dans l'interpellation, il y a engagement, duel des regards. Regard/regardé.

Dans Goldberg: Variations, le côté statique de l'image fixe est rendu par des verbes de stase, par des passifs, par l'énumération, la juxtaposition, la parataxe, le lexique de la fixité. L'artifice (au sens noble de création non naturelle) de l'image (peinte ou autre) fait que tout cela est "arrangé » et "disposé", et le texte en rend compte en décrivant l'agencement des objets/sujets, plus que directement (illusoirement) tel personnage, tel placard, censés être présents. Cadrages, composition, lumière qui ne va pas varier, propriétés intrinsèques d'une image, convergent ici pour produire l'impression picturale. Comme dans «l'effet-tableau » ou " l'arrangement esthétique », qui suggère le tableau, une image. Le vocabulaire de l'image, le vocabulaire de la critique, de l'histoire de l'art aussi, infusent le texte et l'affectent d'un coefficient artistique. Ils font appel aux connaissances du lecteur, à la reconnaissance du genre (nature morte), de «trucs " d'ateliers (comme les reflets sur des bouteilles) et de dispositifs, ainsi que de dispositions.

L'étude du panneau décrit par Josipovici montre qu'il s'agissait là d'une œuvre indépendante probablement commandée par un barbier ou un médecin, une Nature morte aux bouteilles et aux livres conservée au musée de Colmar ${ }^{2}$. On a réussi à la dater de 1470, et 
selon Charles Sterling, il pourrait s'agir là de la toute première nature morte de l'art occidental. En outre, l'histoire de l'art permet aussi de corriger une erreur du narrateur de Josipovici, qui a cru voir dans la forme ovale posée sur une boite, un citron : «Balanced on top of [the round box] is the fruit, probably a coarse skinned lemon, the light bringing out its grainy texture " (Josipovici 68). "Probably », dans le passage, indiquait bien le doute. Charles Sterling a montré qu'il s'agissait là non d'un fruit mais d'un bezoar, concrétion qui se trouvait dans l'estomac ou les intestins de mammifères marins. On l'utilisait pour se protéger de toutes sortes de poisons et/ou pour guérir les maladies. C'était un élément fort prisé qui entrait dans les Wunderkammers, souvent montés sur des métaux précieux, et Rodolphe II en possédait un (Sterling 7-17; Falguières 40). Cette Nature morte aux bouteilles et aux livres n'est-elle pas une version sobre et populaire des magnifiques cabinets de curiosités souvent disposés dans de précieuses armoires? La collection a lien avec le temps dont elle aligne les objets qu'elle a recueillis comme en une syntaxe du temps rendu visible, ce qu'a très bien « rendu » Virginia Woolf dans sa nouvelle « Solid Objects ».

21 Pour identifier l'ekphrasis puis le panneau, il s'agissait donc pour le lecteur d'être disponible, sensible à l'impression qui se lève, à sa confirmation (ou infirmation), voire à une impression, fugitive comme un voile ou un nuage qui passe sur la page entre les signes lus et l'imagination du lecteur. La combinaison entre texte et image qui donne temps à l'image, et combine l'immobilité et le vif du temps vécu dans une durée textuelle, est paradoxale mais indéniable. Elle réalise «l'immobilité vive» et relève de ce que je propose d'appeler « le moment ekphrastique ».

\section{« Le moment ekphrastique » : la « dis-tempsion»}

Le «moment ekphrastique » est ce moment du texte occupé par l'ekphrasis, et qui a des résonances dans un avant et un après du texte narratif en prose ou en poésie narrative (celle de Browning ou de Tennyson par exemple) - ce que l'on peut nommer une opération de «dis-temps » : une ouverture du temps dans sa durée, lorsque le temps est distendu / « dis-temps-du ». Wendy Steiner a utilisé le terme sans autre précision (Steiner 288). Je souhaite lui donner un poids théorique plus lourd en le forgeant à partir du "moment fécond» ("pregnant moment», kairos), moment choisi comme sujet de l'ekphrasis mais défini comme justement court, figé et particulièrement représentatif d'une action plus longue. Or, entre le moment étendu de l'action entière et le (plus ou moins) court «moment» de l'ekphrasis, on peut déceler une autre modalité temporelle, comme un changement de régime ou de vitesse, celle-ci étant un rapport entre le temps et l'espace. Il s'agirait d'une "durée ekphrastique», comme distendue (entendre "distemps-due »), préparée par le texte, et qui retentit encore après son arrêt, comme autant de ronds dans l'eau après la chute d'un caillou. Ce moment a un retentissement par ondes qui permet d'étendre l'effet de l'ekphrasis sans la réduire au(x) paragraphe(s) de description. Il s'agit de prendre en compte sa ou ses résonance(s). Le "moment ekphrastique " permet d'inscrire le visuel dans la tradition du choix pictural ou photographique. Il permet ensuite de situer l'ekphrasis sur l'axe du temps - ce que Lessing refusait -, de montrer aussi l'ambiguïté de ce genre entre-deux, à la limite de deux arts. Le "moment ekphrastique » est aussi un moment de risque, un moment où les deux arts se testent l'un par rapport à l'autre sans affrontement mais aux limites de leurs capacités. Leur friction ou grésillement est alors riche de potentialités. 

spectateurs dans la diégèse tout comme sa description tient en haleine le lecteur. Il fait mine d'être vivant/transparent alors même que l'immobilité règne pour que l'on puisse admirer à loisir le spectacle sans qu'il n'y ait aucun trouble du bougé de la présence vibrante. Car sous couvert de montrer/exhiber (un tableau, une femme, une femme en son tableau), il recouvre sous des mots (ensevelit) la forme de cette femme, objet de convoitise. L'ekphrasis, parce qu'elle séduit et maintient sous son charme, entre en tension avec sa fonction maïeutique de révélation. Frêle rideau de chair, diaphane de l'illusion... à cause justement de cet avant et cet après qui étirent et construisent ce «moment ekphrastique » du texte, cet « avènement » du tableau-vivant dans l'histoire.

\section{Un événement de lecture}

L'entre-deux qui fait advenir le tiers est un «événement » du vif, du mouvement produit par ce passage entre deux media. Il relève de ce que Louis Marin appelait aussi un " événement de lecture ", cet événement "au sens très humble de ce qui advient, de ce qui arrive dans ce qu'on lit, sans s'annoncer, presque imprévisible " (Marin 15). Quand l'image advient au texte, elle le rompt, l'interrompt, le disjoint, « objecte » et provoque une bifurcation et un flottement, un tremblement. À cause de son hétérogénéité, on ne peut l'ignorer. Cela peut provoquer un choc ou au moins faire obstacle entre le texte et l'œil interne du lecteur. En tant qu'objet visuel, il s'inter-pose, subvertit, fait tressaillir, transmet son énergie. Il ne s'agit pas seulement d'un tiers qui rendrait possible le passage et serait au service des deux autres arts. C'est un actant à part entière de la relation intermédiale, le lien nécessaire et pourtant virtuel, le moment de l'image.

On peut donc accepter l'idée que la lecture du texte/image produit un événement, et un événement "c'est ce qui vient " pour Derrida, soit «l'irruptif, inaugural, singulier " (Derrida 61), si on ne le voit pas venir. Un avènement aussi. Comment le lecteur est-il affecté, ou " pointé » (Barthes) par le texte/image ? Il y a du ressenti là-dedans puisque le corps est en jeu. Que se passe-t-il : une ouverture? une rêverie aussi ? un tressaillement, une émotion? Le lecteur, stimulé, alerté, délogé, devient actif : il opère superpositions, surimpressions, collages, voire un montage qui rend l'image dialectique. Le texte/image implique forcément une co-production. Une «mise en mouvement du livre» (Zimmermann 110). Le texte alors se fait « œuvre biface», polymorphe, il est le résultat d'une coopération aussi. Derek Attridge reconnait l'actualisation nécessaire : «c'est en le lisant qu'un texte devient de la littérature $»^{3}$, comme une venue à la vie du texte « contenu » entre les couvertures du livre et qui n'existe pas hors de la lecture.

On pourrait donc aussi envisager l'effet du texte/image comme une question de retentissement et le dictionnaire Robert rappelle que re-tentir vient du latin populaire " tinnitire », « tinnire », qui signifie « résonner ». Le premier sens du mot est : « être ébranlé, rempli par un bruit, un son fort et aussi se faire entendre avec force. Didactiquement: exercer une action, avoir des répercussions, un retentissement sur ». Événement, surgissement dynamique, il y a là aussi quelque chose de l'ordre de l'esthétique de la surprise (du plissement aussi), de l'inattendu en tout cas, qui provoque une recomposition, une modification de l'existant. Il bouleverse et entraîne un réexamen de ce que l'on tenait pour vrai, pour stable, une re-disposition, un ré-agencement. Utile, car il remet en jeu, il porte à la question. C'est là l'une des fonctions du visuel en texte, l'un de ses enjeux éthiques : provoquer le plissement de l'interrogation, le dé-rangement de 
l'événement, par la traversée réciproque des deux arts. Quand l'art « objecte " (Jeanette Winterson, Art Objects, 1995) à sa manière. "Or la manière est image " rappelle Nancy dans $\mathrm{Au}$ fond des images. "Elle est ce qui fait image, y compris dans le texte. Faire image, c'est donner du relief, du saillant, du trait, de la présence. L'image avant tout donne présence. C'est manière de présence. Manière et matière de présence. On l'a souvent dit : aucun discours ne peut rivaliser avec la puissance d'une image. (Reste que discours n'est pas texte) » (Nancy 125). Présence de l'image retournée comme un gant vers le texte. Une immobilité vive en effet.

Se produit alors comme un affect sous le coup de la libération des forces capturées dans l'œuvre, provoquant aussi une expérience et une pensée singulières de l'œuvre, comme Laurent Jenny a pu parler de "parole singulière" ou encore Derek Attridge de La Singularité de la littérature. Quelque chose " arrive " par la sensation dans la sensation. Les résonances sensibles tirent l'individu vers le singulier, vers les vibrations de ce que j'ai appelé ailleurs l'iconorythme (voir Louvel, Textes/Images), mélange de texte/image, de temps et de vitesse. Le mouvement du voir donne au texte le rythme du tableau qui se combine à celui de la lecture. Et Nancy de s'interroger :

Mais qu'est-ce que donner présence ? N'est-ce pas donner ce qui n'est pas à donner : ce qui est ou n'est pas [...] C'est la quadrature du cercle ou bien celle de l'amour, qui donne ce qu'il n'a pas à qui n'en veut pas, selon le mot d'un analyste, c'est-à-dire d'un spécialiste des images-textes. L'image donne une présence dont elle est dépourvue - n'ayant d'autre présence irréelle que sa minceur pelliculaire - elle la donne à ce qui, étant absent, ne saurait la recevoir. (Nancy 126)

Saisir l'œuvre et être saisi par elle lorsque l'on est véritablement "touché », accepter aussi d'être dessaisi pour mieux voir, une question de prise et de déprise, voilà ce que met en vibration le texte/image.

\section{Du côté de la réception}

C'est que l'approche sensible a une résonance singulière et d'abord permet de libérer les affects. L'image serait-elle alors, dans certains cas, davantage médiatrice d'impressions que modèle d'écriture, moyen d'accéder à des vérités autrement inouïes, impossibles à dire. Moyen d'approcher « la vraie vie » et d'en faire apparaître l'essence, de retisser les liens avec la mémoire aussi, comme c'est le cas chez Proust. L'image pourrait procurer un apaisement pour Lacan, on l'a vu, tout en étant un "piège à regard", dans le cas de l'anamorphose. Du moins sa contemplation provoquerait une « saisie » du sujet. Certaines images, au contraire, libèrent des forces sidérantes et bouleversantes: les tableaux de Bacon suscitent un émoi difficilement supportable à haute dose, comme lors d'une rétrospective, par exemple. On est peut-être alors sur le régime de la phrase-affect définie par Lyotard dans Le Différend. En tout cas, quels qu'ils soient, les tableaux, photographies, produisent des affects. Le corps est touché, « saisi » par l'image, dans sa rencontre avec elle. Il résonne. L'art saisit le vif.

Il ne s'agit pas ici d'entrer dans les discussions qui divisent cognitivistes, psychanalystes et neurologues au sujet des images mentales (voir Tisseron 127) ou des émotions, à la suite des travaux d'Antonio Damasio, par exemple, mais simplement de les signaler tant elles sont riches d'enseignements. Eric Laurent présente la position de l'analyste au sujet de la discussion qui séparerait l'émotion affectant le corps, du sentiment qui serait de l'ordre de la cognition, de l'intellect (Laurent 99-123)4. Lionel Naccache, en tant que neurologue, explore ce qu'il nomme "le nouvel inconscient», et met en regard la 
psychanalyse (l'inconscient freudien) et la cognition (l'inconscient cognitif) ainsi que les questions de représentation mentale et de conscience, à la lumière d'expériences récentes des neurosciences de l'esprit ${ }^{5}$. La piste cognitiviste reste à explorer en ce qui concerne les études intermédiales. Ce pourrait être une étape prochaine, un champ ouvert à d'autres idées de recherche. Tout comme le destin de l'image dans un monde où l'électronique règne.

\section{Le tiers pictural : l'événement entre-deux}

Le pictural (au sens de " picture ", d'image) mis en mots peut servir à rendre compte de la tension du texte lorsqu'il est traversé par l'image, est in-formé par elle. Tiraillé entre l'œil et l'oreille, la stase et le vif, sa figure en est l'oxymore doublé de synesthésie, pour faire bonne mesure. Il est ainsi doté d'une qualité visuelle (artistique, optique, photographique, etc.) qui outre son énergie et ses forces propres, outre l'intensité qu'il dégage, a aussi du sens, un mode de signification autre dont on ne peut faire l'économie. Le passage entre deux media se lit entre-deux, le lecteur n'étant jamais totalement dans l'un, ni totalement hors de l'autre. Cette instabilité du texte/image, son oscillation sans fin, qui résulte de la mise en rapport du texte et de l'image, du temps et du visuel, fascine l'écrivain et le lecteur car elle les loge constamment dans la transposition, la trans-action, la négociation, «le change du rapport ». J'ai donc proposé de nommer « tiers pictural » cet événement, cet entre-deux, tiers nécessaire pour analyser un certain type de textes à fort coefficient pictural. Je le construis sur le «tiers instruit» de Michel Serres et sur «le troisième livre » de Derrida parlant de Jabès, et du livre qui reste en suspens quelque part, entre celui que le lecteur tient entre les mains et celui que l'auteur a voulu, un supplément, une « invention » dans tous les sens du terme.

Le tiers pictural est cet entre-deux vibrant entre texte et image, à l'instar de la barre oblique qui séparerait les deux. Moyen terme, il est une activité qui se joue entre-deux. C'est ce qui s'actualise sur l'écran de l'œil intérieur du lecteur-spectateur. Dans ces «machines obscures" (Noël 19) que nous sommes, joue la dynamique du tiers pictural: mouvement, énergie qui entraîne une perturbation, un surplus de sens et d'affect, une rêverie qui danse entre les deux. Ni l'un ni l'autre, il est l'un et l'autre en tours et retours de l'image. Il s'agit vraiment d'une modalité qui est de l'ordre du vivant, du mouvement, du désir, de l'expérience ressentie, de l'événement au sens de ce qui advient: une opération aussi, une performance. Pas si immobile donc!

Le « tiers pictural » entre le texte et l'image, fait advenir autre chose, ce qui joue entre les deux dans le vif du sujet. Il serait l'image flottante (virtuelle ou «réelle» au sens de Descartes, une « image en l'air » selon Agnès Minazzoli (84), image " flottante ») suggérée par le texte mais qui reste une image suscitée par des mots, une image qui peut renvoyer à une image réelle ou imaginaire à reconstruire par le lecteur, image qui sera alors sa propriété, son «invention" (sa "découverte»), puisqu'elle ne coïncidera jamais avec celle qui fut mise en texte par le narrateur plongé dans sa vision intérieure. Elle fait bouger les limites, vaciller les certitudes.

Le tiers pictural serait donc sur un écran virtuel le moment où se jouerait le petit drame du montré-caché, comme dans "le tableau vivant», où la chair apparaissait sous le maillot troublant, une pulsation, diastole et systole, apparition/disparition, l'aphanisis/ epiphasis qu'évoque Georges Didi-Huberman (26 et 47-48). La référence picturale montre et masque. Voile discursif, elle provoque une excitation, suscite le désir. L'immobilité 
devient vive quand le vif «joue» l'immobile de l'œuvre, quand l'œuvre se fait sous nos yeux aussi, une venue au monde. Une création, mieux une cré-action. C'est bien aussi ce que met en scène la longue élaboration du tableau éponyme dans La Jeune Fille à la perle de Tracy Chevalier (1999), tout comme c'était le cas dans Le Chef d'œuvre inconnu de Balzac, dans L'Cuvre de Zola et dans tant d'autres...

\section{BIBLIOGRAPHIE}

Barthes, Roland. La Chambre claire. Paris : Gallimard/Cahiers du Cinéma/Éditions du Seuil, 1980.

Benjamin, Walter. «L'œuvre d'art à l'ère de sa reproductibilité technique ». Essais $2:$ 1935-1940. Paris : Denoël/Gonthier, 1983.

Borel, France. Le Modèle, ou l'artiste séduit. Genève : Éditions Skira, 1990.

Derrida, Jacques. Penser à ne pas voir. Paris : La Différence, 2013.

Didi-Huberman, Georges. La Peinture incarnée. Paris : Éditions de Minuit, 1989.

Falguières, Patricia. Le Maniérisme : une avant-garde au XVI siècle. Paris : Gallimard, « Découvertes », 2004.

Heffernan, James. Museum of Words: The Poetics of Ekphrasis from Homer to Ashberry. Chicago: Chicago UP, 2004.

Josipovici, Gabriel. Goldberg: Variations (2002). New York: HarperCollins, 2007.

Laurent, Eric. Lost in Cognition, Psychanalyse et sciences cognitives. Nantes : Éditions Cécile Defaut, «Psyché », 2008.

Louvel, Liliane. Textes/images. Rennes : PUR, 2002.

Lyotard, Jean-François. Le Différend. Paris : Éditions de Minuit, 1983.

Marin, Louis. L'Écriture de soi. Ignace de Loyola, Montaigne, Stendhal, Roland Barthes. Paris : PUF, 1999.

Minazzoli, Agnès. La Première Ombre. Paris : Éditions de Minuit, 1989.

Naccache, Lionel. Le Nouvel Inconscient. Paris : Odile Jacob, 2006.

Nancy, Jean-Luc. Au fond des images. Paris : Galilée, 2003.

Noël, Bernard. Journal du regard. Paris : POL, 1988.

Steiner, Wendy (ed.). Art and Literature 1, Poetics Today 10:1 (1989): 279-297.

Sterling, Charles. La Nature morte de l'Antiquité à nos jours. Catalogue de l'exposition à l'Orangerie des Tuileries, 1952.

Tisseron, Serge. «L'image comme processus, le visuel comme fantasme ». Cahiers de psychologie clinique 20 (2003/1): 125-135.

Taylor-Wood, Sam. A Little Death. Youtube:

https://www.youtube.com/watch?v=H21ypJb7KJk

(dernière consultation 01/10/2017) 
Taylor-Wood, Sam. Still Life. YouTube:

https://www.youtube.com/watch?v=BJQYSPFo7hk

(dernière consultation 01/10/2017)

Taylor-Wood, Sam. David Beckham Sleeping. YouTube:

https://www.youtube.com/watch?v=loGPpkWEpdU

(dernière consultation 01/10/2017)

Wajcman, Gérard. Fenêtre: Chronique du regard et de l'intime. Paris : Verdier, « Philia », 2004.

Zimmermann, Laurent. Penser par les images. Autour des travaux de Georges Didi-Huberman. Paris :

Cécile Defaut, 2006.

\section{NOTES}

1. Seconde erreur dans le même texte : le drapeau italien, dont Barthes dit qu'il est rouge jaune et vert, erreur que mes étudiants rectifient aussitôt...

2. http://www.musees-alsace.org/de/sammlungen/werk/?ref=504000032_nature-morte-auxbouteilles-et-aux-livres (dernière consultation le 01/10/2017).

3. Derek Attridge, table ronde sur "La littérature et ses enjeux », ESSE-9 Conference, Aarhus Denmark, août 2008.

4. Laurent discute des thèses d'Antonio Damasio avec l'aide de celles de Bernard Victorri (chapitre 3, « Psychanalyse et cognition »).

5. Lionel Naccache est neurologue et travaille à l'hôpital de la Pitié-Salpétrière à Paris. Il est également chercheur en neurosciences cognitives au sein de l'unité Inserm Neuro-imagerie cognitive. Je remercie P. Ortel de m'avoir signalé cet ouvrage à l'issue d'une conférence que j'ai donnée à l'Université de Toulouse-le-Mirail.

\section{RÉSUMÉS}

«L'immobilité vive» que l'on nous donne à penser repose sur un oxymore, un paradoxe, l'alliance de contraires habituellement opposés comme dans la dichotomie, classique au sein des études artistiques, entre la vie et la mort, l'amour et la haine, le temps et l'espace. Une alliance de contraires, ce qui n'est pas le cas du texte et de l'image mis ensemble, puisque l'oscillation constitutive du rapport texte/image est aussi le lieu de l'émergence du «tiers pictural » (Louvel), cette apparition entre deux de l'image comme « une petite étoile à la vitre du texte ». Ce qui nous rappelle le punctum barthésien. On ira donc voir du côté de la nature morte qui, bien qu'immobile, peut s'animer, still life à la Taylor-Wood, ou du côté d'un texte de Gabriel Josipovici, qui met en mots une nature morte. On verra comment le moment ekphrastique constitue une dis-tempsion du texte pris entre fixité et progression. C'est le lieu de «l'événement de lecture " (Louis Marin) quand l'image, tout à coup, se lève d'entre les lignes, provoquant l'effet $\mathrm{du}$ « tiers pictural ", matière de présence.

The "intense immobility" we have to think and rethink here rests on an oxymoron, the combination of opposites which are usually pitted one against the other in artistic studies, as in 
life and death, love and hate, time and space. A combination of opposites, which is not what the relation between word and image is when they work together, since the oscillation of the word/ image relation also is the locus of the "pictorial third" (Louvel). This results from the in-between appearance of the image causing "a small star on the window pane of the text", reminding us of Barthes's punctum. It will be necessary to revisit the genre of still lives which, though immobile, may be endowed with movement, as is the case with Sam Taylor-Wood's videos. Or with one of Gabriel Josipovici's novels which gives pride of place to a still life painting. It will be easy to see then how the ekphrastic moment operates a dis-timing of the text caught-up between stillness and motion. This is the moment when the "reading event" (Louis Marin) happens to the reader, when an image abruptly rises from between the lines, producing "the pictorial third", the very stuff which visual presence is made of.

\section{INDEX}

Mots-clés : nature morte, oxymore, événement de lecture, tiers pictural, ekphrasis, réception, présence, intensité

Keywords : still life, oxymoron, reading event, pictorial third, ekphrasis, reception, presence, intensity

\section{AUTEUR}

\section{LILIANE LOUVEL}

Liliane Louvel est professeur émérite de littérature britannique à l'Université de Poitiers. Son champ de recherche est la littérature britannique contemporaine et les rapports texte/image. Elle a publié cinq ouvrages sur les rapports entre texte et image : L'Eil du texte (Toulouse : PUM, 1998), The Picture of Dorian Gray, le double miroir de l'art (Paris : Ellipses, 2000), Texte/image, images à lire et textes à voir (Rennes : PUR, 2002), Le Tiers pictural, pour une critique intermédiale (Rennes : PUR 2010), Poetics of the Iconotext (trad. Laurence Petit, ed. Karen Jacobs, Ashgate, 2011). Elle a également dirigé ou co-dirigé plusieurs recueils d'essais sur le même sujet : Like Painting... (La Licorne), Actes du colloque de Cerisy : Texte/image nouveaux problèmes (co-éd. Henri Scepi, Rennes : PUR, 1999), Photographie et litterature (co-éd. J.-P. Montier, D. Meaux and P. Ortel, Rennes : PUR, 2008), Carrefour Stieglitz (co-éd. J.-P. Montier \& Jay Bochner, Rennes : PUR, 2012), Intermedial Arts Disrupting Remembering and Transforming Media (co-éd. Leena Leilitta \& Sabine Kim, Cambridge Scholars Press, 2012), Musing in the Museum (co-éd. Laurence Petit \& Karen Brown, Word/Image, Taylor and Francis, May 2015). Le Tiers pictural, traduit par Angeliki Tseti, sera publié en 2018 chez Routledge. Elle est actuellement présidente de ESSE (European Society for the Study of English), et de IAWIS/IAERTI (International Society of Word \& Image Studies). 